\title{
Effects of quetiapine and olanzapine in patients with psychosis and violent behavior: a pilot randomized, open-label, comparative study
}

This article was published in the following Dove Press journal:

Neuropsychiatric Disease and Treatment

7 May 2014

Number of times this article has been viewed

\section{Gabriella Gobbi ${ }^{1,2}$ \\ Stefano Comai' \\ Guy Debonnel $1,2, \uparrow$}

'Neurobiological Psychiatric Unit, Department of Psychiatry, McGill University and McGill University Health Center, ${ }^{2}$ Institut Philippe Pinel, Department of Psychiatry, Université de Montréal, Montréal, QC, Canada

†Guy Debonnel passed away on November 4, 2006
Correspondence: Gabriella Gobbi Neurobiological Psychiatry Unit, Department of Psychiatry, McGill University, 1033 av Des Pins Ouest, Montréal, QC, Canada

$\mathrm{Tel}+$ I 5I4 398 I290

Fax + I 5 I4 3984866

Email gabriella.gobbi@mcgill.ca
Objective: Patients suffering from psychosis are more likely than the general population to commit aggressive acts, but the therapeutics of aggressive behavior are still a matter of debate. Methods: This pilot randomized, open-label study compared the efficacy of quetiapine versus olanzapine in reducing impulsive and aggressive behaviors (primary endpoints) and psychotic symptoms (secondary endpoints) from baseline to days 1, 7, 14, 28, 42, 56, and 70, in 15 violent schizophrenic patients hospitalized in a maximum-security psychiatric hospital.

Results: Quetiapine $(525 \pm 45 \mathrm{mg}$ ) and olanzapine $(18.5 \pm 4.8 \mathrm{mg})$ were both efficacious in reducing Impulsivity Rating Scale from baseline to day 70. In addition, both treatments reduced the Brief Psychiatric Rating Scale, Positive and Negative Syndrome Scale, and Clinical Global Impression Scale scores at day 70 compared to baseline, and no differences were observed between treatments. Moreover, quetiapine, but not olanzapine, yielded an improvement of depressive symptoms in the items "depression" in Brief Psychiatric Rating Scale and "blunted affect" in Positive and Negative Syndrome Scale. Modified Overt Aggression Scale scores were also decreased from baseline to the endpoint, but due to the limited number of patients, it was not possible to detect a significant difference.

Conclusion: In this pilot study, quetiapine and olanzapine equally decreased impulsive and psychotic symptoms after 8 weeks of treatment. Double-blind, large studies are needed to confirm the validity of these two treatments in highly aggressive and violent schizophrenic patients.

Keywords: schizophrenia, aggression, violence, impulsivity, atypical antipsychotics, depressive symptoms

\section{Introduction}

Mentally ill patients present an elevated rate of aggressive behaviors. Psychotic patients are five times more likely than the general population to commit violent acts, defined as "physical attacks on other persons, or on one's self (self mutilation), with deliberate destructive intent sufficiently severe to justify a legal measure". ${ }^{1}$ Impulsivity is a factor strictly linked to aggression which, when present, increases the risk of aggressive behavior., ${ }^{2,3}$

Aggression is a complex phenomenon associated with genetic, neurobiological, and psychosocial factors. ${ }^{4}$ Impairments of many neurotransmitter systems, including the serotonin (5-HT), dopamine (DA), and norepinephrine (NE) systems, are implicated in the biology of aggression. ${ }^{5,6}$ The most consistent neurobiological data on the pathogenesis of aggression and impulsivity concern modifications of serotonergic (5-HT) neurotransmission. ${ }^{7}$ For example, several studies have reported that violent and suicidal patients have attenuated levels of 5-hydroxyindoleacetic acid (5-HIAA), the primary 
metabolite of 5-HT, in their cerebrospinal fluid, ${ }^{8-12}$ although many studies have also failed to find an association between aggression and low cerebrospinal fluid 5-HIAA levels. ${ }^{13}$ Depletion of tryptophan, which in turn reduces central 5-HT levels by decreasing the levels of available precursor, also increases violent and impulsive behaviors. ${ }^{14-17}$

Clozapine was the first atypical antipsychotic agent for which clear anti-aggressive properties were demonstrated. ${ }^{18-28}$ Because clozapine therapy can result in the development of agranulocytosis ${ }^{29}$ and frequent blood tests are required to avoid such complications, clozapine may not be an optimal treatment, particularly in aggressive patients for whom collecting blood samples on a regular basis is not an easy task. Olanzapine, an atypical neuroleptic ${ }^{30}$ which, similarly to clozapine, shows high affinity for both the $5-\mathrm{HT}_{2 \mathrm{~A}}$ and the $5-\mathrm{HT}_{2 \mathrm{C}}$ receptors, ${ }^{31}$ has also demonstrated efficacy in reducing aggressive behaviors in psychotic patients. ${ }^{32-35}$

Quetiapine's unique binding profile includes partial agonist action at 5-HT1A receptors ${ }^{36}$ and low-affinity antagonist action at 5-HT2A receptors, ${ }^{37}$ along with a lack of action at 5 -HT2C receptors. ${ }^{38}$ Considering these effects on the serotonergic system, it is logical to hypothesize that quetiapine should have an effect on the control of aggression and impulsivity. Indeed, several randomized, double-blind, placebo-controlled studies have demonstrated that quetiapine therapy effectively attenuates aggressive behavior in patients suffering from schizophrenia. ${ }^{39-42}$

Only a few studies have compared the effect of olanzapine and quetiapine in patients suffering from schizophrenia over short-term ${ }^{43}$ and long-term ${ }^{44,45}$ periods. Moreover, none of these studies were carried out in selective violent patients. Due to the shortage of relevant research, the goal of the present study is to compare the efficacy of olanzapine and quetiapine in treating aggression in patients suffering from schizophrenia. Moreover, unlike previous research, the present study employs a sample of extremely aggressive inpatients from a forensic psychiatric hospital, specifically investigating the drugs in a dangerously violent population.

\section{Methods}

This randomized, single-center, 10-week, open-label study compares the effects of quetiapine and olanzapine in psychotic patients presenting with serious problems related to aggression (Study code: 5077-9056). At the time of the study (January 2003-June 2005), the investigators were not aware that quetiapine possessed antidepressant effects. The primary purpose of the study was to compare the efficacy of quetiapine versus olanzapine in reducing impulsive and aggressive behaviors, measured with the Modified Overt Aggression Scale (MOAS) ${ }^{46}$ and the Impulsivity Rating Scale (IRS), ${ }^{47}$ in psychotic patients. The secondary purpose was instead to compare the efficacy of quetiapine versus olanzapine in reducing the intensity of psychotic symptoms measured with the Brief Psychiatry Rating Scale (BPRS) ${ }^{48}$ the Positive and Negative Syndrome Scale (PANSS), ${ }^{49}$ the Clinical Global Impression-Severity (CGI-S), and the CGI-improvement (CGI-I) scale. ${ }^{50}$ In addition, the possibility of a correlation between the reduction of aggressive behaviors and that of the psychotic symptoms was also assessed.

After being admitted to the study, patients were randomly assigned to either the quetiapine (up to $800 \mathrm{mg} /$ day) or olanzapine (up to $20 \mathrm{mg} / \mathrm{kg}$ ) group for 10 weeks. Random numbers, generated from a set exhibiting statistical randomness, were communicated by an independent statistical center to the research nurse. This study included only inpatients treated for schizophrenia, schizoaffective disorder, or paranoid disorder.

\section{Patients}

Thirty patients were screened and a total of 15 participants were then selected for the study. The patients were inpatients at the Philippe Pinel Institute, a maximum-security Canadian psychiatric hospital (300 beds) located in Montreal, Québec, specialized in the treatment and evaluation of patients suffering from psychiatric diseases and showing aggressive or impulsive behavior. These patients come from other psychiatric hospitals, prisons, or other institutions of the Ministry of Health and Social Service of Québec. The study was originally designed to enroll 20-25 patients per group in order to have a power of 0.8 at an alpha level of 0.05 . Due to the difficulty in the recruitment process (see Discussion), we then decided to carry out only a pilot study. The study was approved by the Philippe Pinel Institute Ethical Committee and was conducted according to the ethical principles for medical research involving human subjects as proposed by the World Medical Association Declaration of Helsinki. All patients signed an informed consent form before participating in the study.

\section{Inclusion and exclusion criteria}

Eligible patients were between 18 and 65 years of age and had a primary diagnosis of schizophrenia, schizoaffective disorder (any subtype), or paranoid disorder according to the Diagnostic and Statistical Manual of Mental Disorders, 4th edition, Text Revision (DSM-IV-R) criteria. A score of at least 15 on the positive scale of the PANSS was also required for study enrollment, and eligible patients had to have been 
considered as having a specific problem related to aggression or impulsivity. Finally, eligible patients had to have been receiving a stable daily dose (within approximately $20 \%$ of the current dose) of a single antipsychotic agent for the month prior to the study; patients receiving depot medication were also considered for the study, as long as their last dose of depot was administered at least 2 weeks or one treatment cycle prior to study entry, with no other antipsychotic agent administered during this time.

Principal exclusion criteria were acute exacerbation of the psychotic state having resulted in a recent modification of the antipsychotic medication; concurrent treatment with antipsychotic agents after randomization; treatment with an investigational agent within the previous month; diagnosis of substance abuse using DSM-IV criteria within the previous 3 months; organic mental disease, including mental retardation, rendering the response to investigators unreliable; history of psychosurgery; history of clinically significant physical illness or abnormal laboratory values at screening; inability to give informed consent; pregnancy; and female subjects of childbearing potential without adequate contraception (hormonal contraception, double-barrier methods, intrauterine devices, tubal ligation). Other clinical exclusion criteria were clinically significant electrocardiography abnormality at screening; a QTc greater than 450 milliseconds or administration of medications that prolong the QT interval; known sensitivity to quetiapine or olanzapine or unsuccessful treatment with adequate doses (similar to those used in the present protocol, for at least 4 weeks) of quetiapine or olanzapine during the previous 3 months; diagnosis of any clinically meaningful, unstable renal, hepatic, cardiovascular respiratory, cerebrovascular disease, or other serious, progressive physical disease; any clinically meaningful abnormal finding uncovered during physical examination and/or clinically significant abnormal laboratory results at screening; history of neuroleptic malignant syndrome; use of potent cytochrome P450 inhibitors during the 14 days preceding randomization (ketoconazole, itraconazole, fluconazole, erythromycin, clarithromycin, troleandomycin, indinavir, nelfinavir, ritonavir, and saquinavir), and use of potent cytochrome P450 inducers (eg, phenytoin, carbamazepine, barbiturates, rifampin, and glucocorticoids) during the 14 days preceding randomization. Patients deemed unable or unlikely to follow the study protocol were also excluded from the study.

\section{Scales}

Patients were rated by a psychiatrist for aggression (MOAS), impulsivity (IRS), and psychotic symptoms (BPRS, CGI-S, and CGI-I) at baseline and days 1, 7, 14, 28, 42, 56, and 70. In addition, the PANSS scale was completed at baseline and days $1,28,42,56$, and 70 .

\section{Drugs posology Quetiapine}

Quetiapine treatment was initiated at a dosage of $50 \mathrm{mg}$ twice daily for days 1 and 2, then increased to $100 \mathrm{mg}$ twice a day (bid) on day 3, $150 \mathrm{mg}$ bid on day 4, and $200 \mathrm{mg}$ bid on day 5 . The dosage was subsequently adjusted on the basis of clinical response; three daily dosage levels were allowed: $200 \mathrm{mg}, 300 \mathrm{mg}$, and $400 \mathrm{mg}$. The maximal total daily dose of quetiapine allowed was $800 \mathrm{mg} /$ day.

\section{Olanzapine}

Olanzapine was initiated at a dosage of $2.5 \mathrm{mg}$ at bedtime for days 1 and 2, and was increased to $5 \mathrm{mg}$ on day $3,7.5 \mathrm{mg}$ on day 4 , and $10 \mathrm{mg}$ on day 5 . The dosage of olanzapine was subsequently adjusted on the basis of clinical response between three daily dosage levels: $10 \mathrm{mg}, 15 \mathrm{mg}$, and $20 \mathrm{mg}$. The maximal total daily dose of olanzapine allowed was $20 \mathrm{mg}$ /day. The maximal doses of quetiapine and olanzapine followed the recommendation by Health Canada at the time of the study.

\section{Other medications}

Benzodiazepines were administered when necessary, at the investigator's discretion, during the course of the study. Patients taking typical antipsychotics were also allowed to enter the study if the dosage was stable over the 3 months prior to the study.

\section{Statistical analyses}

Statistical analyses were carried out using the Predictive Analytical Software (PASW; v18; SPSS Inc., Chicago, IL, USA) and Sigma Plot (v12; SPSS Inc.). Numerical values are presented as mean \pm standard deviation (SD) unless otherwise specified. Group comparison on baseline demographic and clinical characteristics was performed using Student's unpaired $t$-test for continuous variables and chi-square test for categorical variables to test lack of balance between groups. MOAS score differences between treatments and time of treatment (baseline and endpoint) were analyzed using Wilcoxon ranksum test. For all the other scales, score changes between treatments and time were evaluated using the two-way repeated measure ANOVA (factors treatment and time) followed by Bonferroni post-hoc test for multiple comparisons. Statistical significance was set at an alpha of 0.05 (two-sided). 
Efficacy analyses were performed using an intent-to-treat data set and an evaluable data set. The intent-to treat data set included all patients who received the treatment but who missed one, two, or three evaluation points. To address missing evaluations, a last observation carried forward (LOCF) analysis was conducted. ${ }^{51}$

\section{Results}

\section{Demographic and clinical characteristics}

The demographic and clinical characteristics of the study population are represented in Table 1. Eight patients were randomly assigned to receive quetiapine and seven to receive olanzapine. The two treatment groups were similar at baseline based on age, sex, diagnosis, and severity of illness (Table 1).

The majority of subjects were male (14 of 15) and Caucasian ( 9 of 15), three patients were Afro-American, two Hispanic, and one American Native. No baseline differences in scores on the MOAS, IRS, BPRS, PANSS positive, or PANSS negative scales between the quetiapine and olanzapine groups were observed (Table 2 and Figure 1). Ten patients received a diagnosis of schizophrenia, as assessed by a qualified psychiatrist following the DSM-IV criteria, while four patients were diagnosed with schizoaffective disorder and one with delusional disorder. The majority of patients (14 out of 15) received a sentence for a crime prior to hospitalization such as murder, attempted murder, or physical or verbal harassment, but were found to be not responsible due to their mental illnesses (these data have not been shown in detail for legal reasons).

Table I Baseline demographic and clinical characteristics of the study population

\begin{tabular}{|c|c|c|c|}
\hline Variable & $\begin{array}{l}\text { Quetiapine } \\
(n=8)\end{array}$ & $\begin{array}{l}\text { Olanzapine } \\
(n=7)\end{array}$ & $\begin{array}{l}\text { Group } \\
\text { comparisons }\end{array}$ \\
\hline Age, mean $\pm S D$, years & $42.88 \pm 10.30$ & $37.57 \pm 9.68$ & $P=0.325^{a}$ \\
\hline Sex, male/female & $\mathrm{I} / 7$ & $0 / 7$ & $P=1.000^{b}$ \\
\hline \multicolumn{4}{|l|}{ Race } \\
\hline Caucasian & 6 & 3 & $P=0.315^{b}$ \\
\hline Afro-American & I & 2 & $P=0.569^{b}$ \\
\hline Hispanic & I & I & $P=1.000^{b}$ \\
\hline American Native & 0 & I & $P=0.467^{b}$ \\
\hline \multicolumn{4}{|c|}{ Psychiatric diagnosis (DSM-IV-R) } \\
\hline Schizophrenia & 5 & 5 & $P=1.000^{b}$ \\
\hline Schizoaffective disorder & 2 & 2 & $P=1.000^{b}$ \\
\hline Delusional disorder & I & 0 & $P=1.000^{b}$ \\
\hline
\end{tabular}

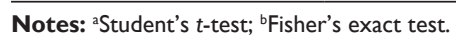

Abbreviations: SD, standard deviation; DSM-IV-R, Diagnostic and Statistical Manual of Mental Disorders, 4th edition, Text Revision.
Table 2 Effects of treatments

\begin{tabular}{|c|c|c|c|c|}
\hline & Baseline & Endpoint & $\mathbf{Z}$ & $P$-value \\
\hline \multicolumn{5}{|c|}{ MOAS - episodes of verbal aggression } \\
\hline \multicolumn{5}{|l|}{ Quetiapine } \\
\hline Mean \pm SD & $2.00 \pm 2.87$ & $1.63 \pm 3.02$ & -0.44 & 0.655 \\
\hline \multicolumn{5}{|l|}{ Olanzapine } \\
\hline Mean \pm SD & $3.14 \pm 5.84$ & $0.00 \pm 0.00$ & -1.66 & 0.102 \\
\hline \multicolumn{5}{|c|}{ MOAS - physical aggression against objects } \\
\hline \multicolumn{5}{|l|}{ Quetiapine } \\
\hline Mean \pm SD & $0.25 \pm 0.70$ & $0.00 \pm 0.00$ & -1.00 & 0.317 \\
\hline \multicolumn{5}{|l|}{ Olanzapine } \\
\hline Mean \pm SD & $0.29 \pm 0.75$ & $0.57 \pm 1.5 \mid$ & -1.00 & 0.317 \\
\hline \multicolumn{5}{|c|}{ MOAS - interventions } \\
\hline \multicolumn{5}{|l|}{ Quetiapine } \\
\hline Mean \pm SD & $0.25 \pm 0.46$ & $0.38 \pm 1.06$ & -1.34 & 0.180 \\
\hline \multicolumn{5}{|l|}{ Olanzapine } \\
\hline Mean \pm SD & $0.7 I \pm I .49$ & $0.14 \pm 0.37$ & -0.44 & 0.655 \\
\hline \multicolumn{5}{|c|}{ MOAS - duration of aggression of total episodes (minutes) } \\
\hline \multicolumn{5}{|l|}{ Quetiapine } \\
\hline Mean \pm SD & $21.25 \pm 34.82$ & $22.50 \pm 44.64$ & 0.00 & 1.000 \\
\hline \multicolumn{5}{|l|}{ Olanzapine } \\
\hline Mean \pm SD & $21.43 \pm 38.59$ & $2.14 \pm 5.66$ & -1.60 & 0.109 \\
\hline
\end{tabular}

Notes: MOAS items score at baseline (day 0 ) and endpoint (day 70 ) in patients treated with quetiapine $(n=8)$ or olanzapine $(n=7)$. Data are expressed as mean \pm SD. MOAS scores presented high variability since they had a minimum value of zero but no upper limit on the maximum value. Consequently, data were not normally distributed and groups were compared using the Wilcoxon rank-sum test.

Abbreviations: MOAS, Modified Overt Aggression Scale; SD, standard deviation.

\section{Medication}

Mean doses at day 7 were $50 \pm 00 \mathrm{mg}$ /day for quetiapine and $2.5 \pm 00 \mathrm{mg} /$ day for olanzapine; at day $28,475 \pm 46 \mathrm{mg} /$ day for quetiapine and $15 \pm 3.2 \mathrm{mg}$ /day for olanzapine; and at day $70,525 \pm 45 \mathrm{mg} /$ day for quetiapine and $18.5 \pm 4.8 \mathrm{mg} /$ day for olanzapine.

\section{Primary scales endpoints: MOAS and IRS}

During the study, no severe episodes of Physical Aggression Against Self, and Physical Aggression Against Other People were reported in the MOAS; for this reason, these two items of the MOAS are not reported in the results. MOAS verbal aggression, episodes of aggression against objects, number of interventions, and the duration of aggression episodes (verbal + against objects) scores were not different comparing baseline versus endpoint (day 70) for both olanzapine and quetiapine (Table 2). Moreover, no differences were detected between the two treatments (data not reported). However, the olanzapine group showed a trend in decreasing the number and duration of episodes of verbal aggression at the end of the treatment.

As showed in Figure 1, the treatment with both quetiapine and olanzapine significantly and equally decreased IRS scores comparing baseline versus endpoint $(P=0.009)$. Indeed, two-way ANOVA for repeated measures indicated 

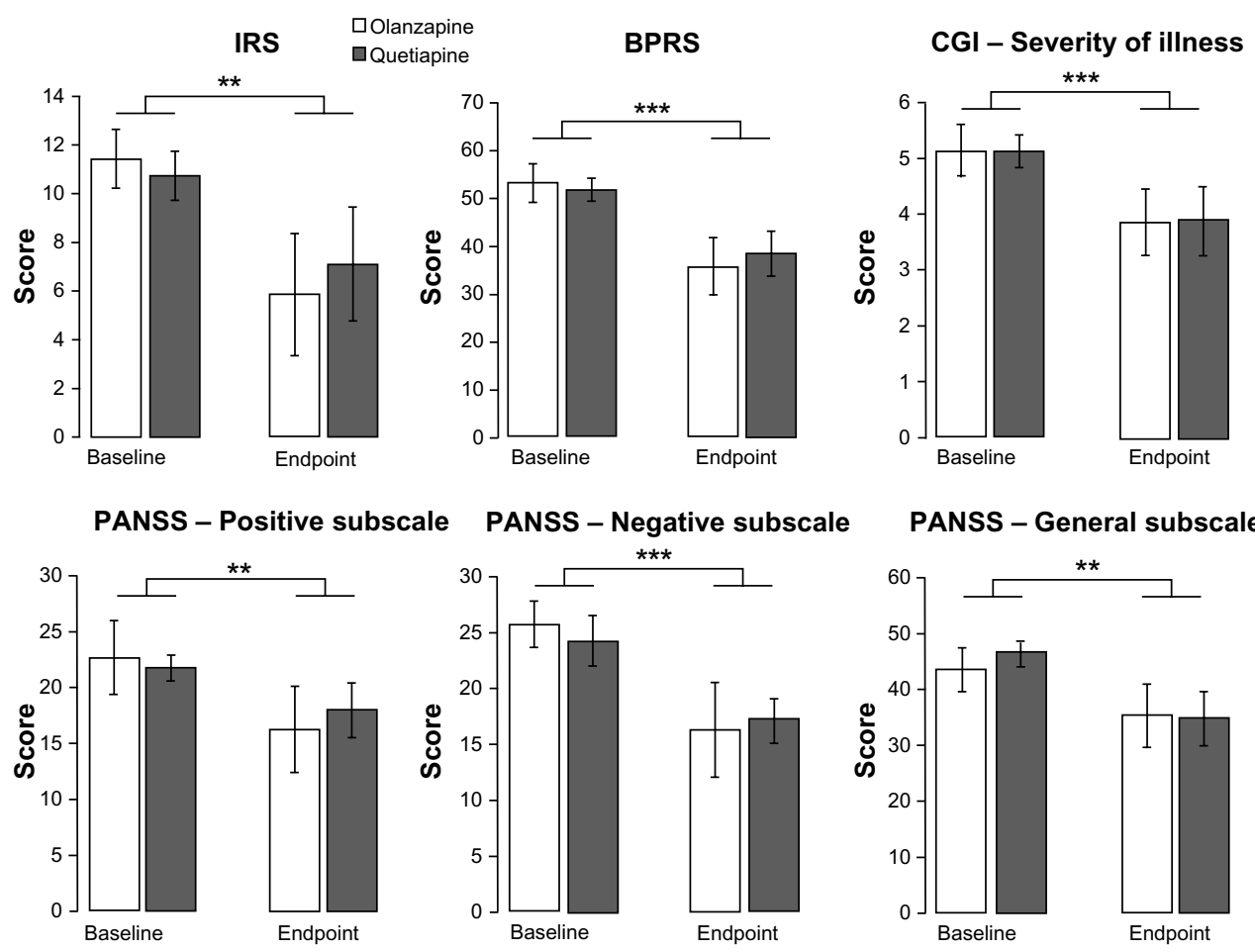

Figure I Effect of treatments. IRS, BPRS, CGI -Severity of illness, PANSS - Positive subscale, PANSS - Negative subscale, and PANSS - General subscale scores at baseline (day 0$)$ and endpoint (day 70$)$ in patients treated with quetiapine $(n=8)$ or olanzapine $(n=7)$.

Notes: Data are expressed as mean $\pm \mathrm{SEM}$; *P $\leq 0.01$; ***P $\leq 0.00 \mathrm{I}$, two-way analysis of variance for repeated measures followed by Bonferroni post-hoc test for multiple comparisons.

Abbreviations: BPRS, Brief Psychiatric Rating Scale; CGI, Clinical Global Impression Scale; IRS, Impulsivity Rating Scale; PANSS, Positive and Negative Syndrome Scale.

an effect of time $\left(F_{1,13}=9.25, P=0.009\right)$ but not of treatment and no interaction between factors. For the quetiapine group, the endpoint was equal to $33.7 \%$ of the baseline score for the IRS; for the olanzapine group, the endpoint was equal to $48.7 \%$ of the baseline score for the IRS.

\section{Secondary scales endpoints: PANSS, BPRS, and CGI}

Two-way ANOVA for repeated measures revealed a significant effect of time for scores on the PANSS General $\left(F_{1,13}=13.61, P=0.003\right)$, Positive $\left(F_{1,13}=9.02, P=0.01\right)$ and Negative $\left(F_{1,13}=18.99, P<0.001\right)$ subscales, as well as BPRS $\left(F_{1,13}=26.35, P<0.001\right)$ and CGI Severity of Illness scale $\left(F_{1,13}=16.48, P=0.001\right)$, all of which decreased over the treatment period (Figure 1). Of note, for all scales, there was no significant effect of treatment and no time $\times$ treatment interaction, meaning that the two treatments produced a similar decrease of psychotic symptoms. For the quetiapine group, the endpoint score was equal to $70.6 \%$ of the baseline score for the PANSS Negative subscale, $82.8 \%$ for the PANSS Positive subscale, $74.9 \%$ for PANSS General subscale, and $75.8 \%$ for the CGI Severity of Illness scale. For the olanzapine group, the endpoint score was equal to $68.3 \%$ of the baseline score for the PANSS Negative subscale, $71.7 \%$ for the PANSS Positive subscale, $81.3 \%$ for PANSS General subscale, and $75.1 \%$ for the CGI Severity of Illness scale.

\section{Scales sub-items: depression}

Using two-way repeated measure ANOVA followed by Bonferroni post-hoc test for multiple comparisons, we looked at possible changes over time and differences between treatments for all the sub-items of the IRS, PANSS, BPRS, and CGI scales. No significant effects were detected except for the sub-items of BPRS (depression) and PANSS (blunted affect) related to depression.

Analysis of the scores on the depression sub-item of the BPRS scale (depression) indicated that quetiapine compared to olanzapine better improved the symptoms of depression at day 70. Indeed, analysis of the changes in BPRS depression sub-item scores between the different time points and baseline revealed an interaction time per treatment $\left(F_{6,78}=2.26\right.$, $P=0.046$ ) and Bonferroni post-hoc analysis for multiple comparisons indicated a difference between olanzapine and quetiapine treatment at day $70(P=0.001$; Figure 2$)$.

Similar results were found for the PANSS sub-item related to depressive mood (blunted affect): there was a significant 

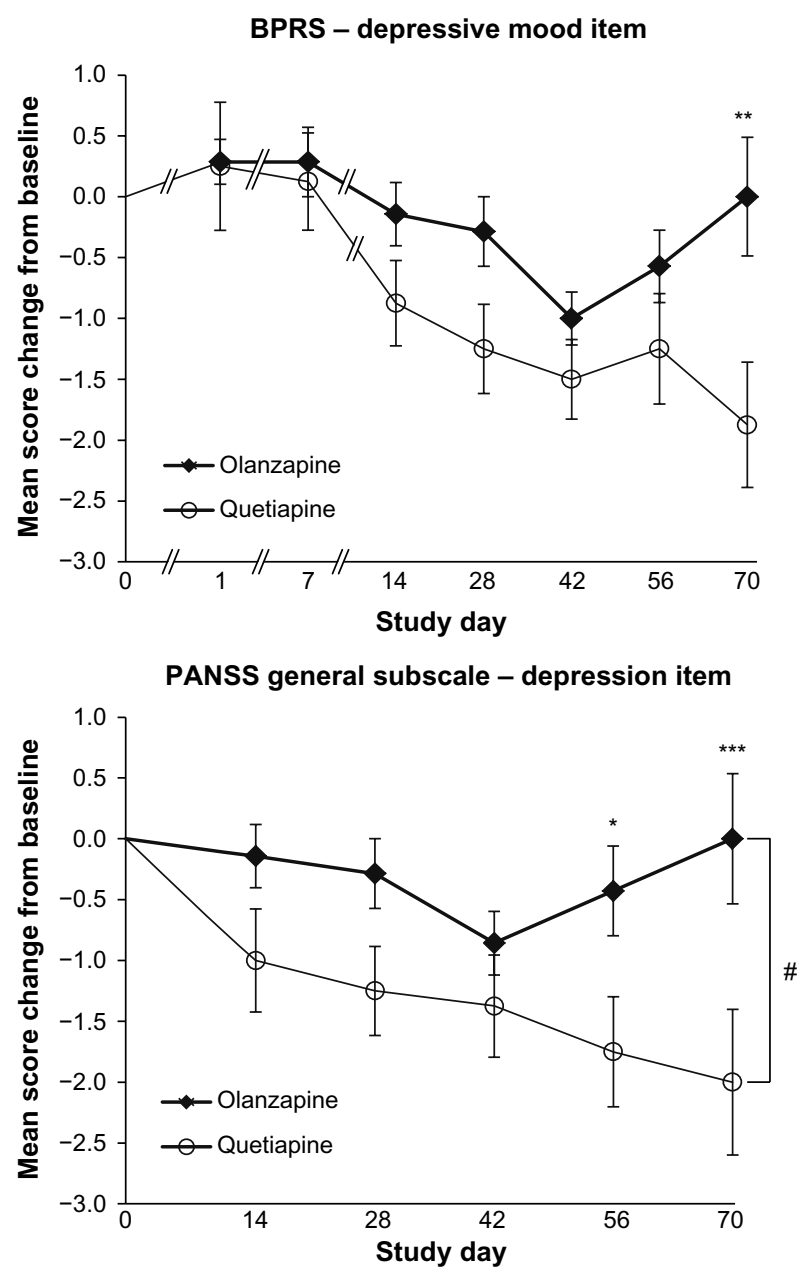

Figure 2 Time course of the depression items mean score change from baseline in the BPRS (top) and PANSS (bottom) in patients treated with quetiapine $(n=8)$ or olanzapine $(n=7)$.

Notes: Data are expressed as mean \pm SEM; $* P<0.05$; $* * P<0.01$; $* * * P<0.00$ I, quetiapine versus olanzapine; ${ }^{\sharp} P<0.05$, effect of treatment; two-way analysis of variance for repeated measures followed by Bonferroni post-hoc test for multiple comparisons.

Abbreviations: BPRS, Brief Psychiatric Rating Scale; PANSS, Positive and Negative Syndrome Scale.

interaction time per treatment $\left(F_{4,52}=2.60, P=0.050\right)$ and post-hoc analysis indicated a difference between olanzapine and quetiapine treatment at day $56(P=0.036)$ and day 70 $(P=0.003$; Figure 2$)$. In addition, there was a significant effect of treatment $\left(F_{1,52}=5.06, P=0.042\right)$.

\section{Metabolic parameters}

Glucose, triglycerides, and HDL-cholesterol values were evaluated at baseline and endpoint. No significant differences in these three parameters were noticed at baseline and endpoint in the olanzapine and quetiapine groups (data not shown).

\section{Discussion}

The results of this pilot study suggest that both quetiapine and olanzapine are effective in improving impulsivity and psychotic symptoms after long-term treatment in a population of criminally violent patients with schizophrenia. We showed that the decrease in impulsivity was paralleled by the decrease in psychotic symptoms for both treatments. Moreover, we found an improvement in depressive items at day 70 after quetiapine but not olanzapine treatment. MOAS scores were also decreased from baseline to the endpoint, but due to the limited number of patients, it was not possible to detect a significant difference.

Remarkably, despite the fact that this randomized clinical trial included a limited number of patients, it was able to detect a beneficial effect of quetiapine and olanzapine treatments for both impulsivity and psychotic symptoms. This study reflects the "real life" in a psychiatric world; in fact the psychotic and violent patients enrolled in the study for 70 days were participating in daily activities despite the high risk of aggression relapse and the consequent safety risk for staff who were in constant contact with these patients. The major drawback of the study was the limited numbers of patients, we could not reach the planned number as indicated in the Methods section, due to the difficulty in keeping these very violent and unpredictable patients in a research protocol for 70 days with limited pharmacological adjustments in case of violent behavior relapse, causing major distress and danger for nurses and staff dealing with these patients.

It is difficult to compare the present research work with that in previous literature due to differences in patient population, scales for evaluation of aggression/impulsivity, and time endpoint.

Three studies have compared the efficacy of quetiapine versus olanzapine in psychotic patients. In one rater-blinded study, 101 acutely psychotic patients were orally administered quetiapine, olanzapine, haloperidol, or olanzapine for agitation; over 72 hours, all four treatments were equally effective in attenuating aggression and hostility scores. ${ }^{43}$ In a prospective, open-label, naturalistic study, 3,135 schizophrenic patients were prescribed olanzapine $(\mathrm{N}=2,118)$, quetiapine $(\mathrm{N}=104)$, clozapine, risperidone, or haloperidol. All treatments decreased the number of patients expressing hostile behavior and this decrease was not significantly different between the olanzapine and quetiapine groups. ${ }^{44}$ In a randomized, double-blind study that was part of the National Institute of Mental Health Clinical Antipsychotic Trials of Intervention Effectiveness (CATIE) project, 1,445 patients with schizophrenia were prescribed either olanzapine, quetiapine, risperidone, ziprasidone, or perphenazine, a typical antipsychotic. ${ }^{45}$ In this study, the olanzapine group ( $\left.\mathrm{N}=185\right)$ experienced an equal reduction in violence risk compared to 
the perphenazine group $(\mathrm{N}=114)$, and the quetiapine group $(\mathrm{N}=131)$ showed a lower risk reduction than perphenazine.

Other studies ${ }^{32,33,52-56}$ have evaluated the effects of olanzapine using the PANSS excited component to evaluate the effects on aggression, reporting a positive effect of olanzapine in the control of extreme behavior, but none of these used validated scales for the measure of impulsivity and aggression.

Krakowski et al, ${ }^{33}$ in a large randomized double-blind study comparing the effect of clozapine, olanzapine, and haloperidol using the MOAS, found that olanzapine decreased verbal and physical aggression, similar to haloperidol, but to a lesser extent than clozapine. Other studies have instead evaluated the anti-aggressive properties of quetiapine using sub-items of the BPRS in schizophrenic patients ${ }^{40,41}$ or PANSS aggression/hostility cluster. ${ }^{42}$

Ganesan et $\mathrm{l}^{57}$ used the Overt Aggression Scale in evaluating aggression in schizophrenic patients in a naturalistic study and found that quetiapine reduced the physical aggression score compared to the other scores by $83 \%$ at day 2 , and that the decrease was maintained until day 5; similar results were also obtained in a small cohort of patients with traumatic brain injury ${ }^{58}$ and adolescents with attention deficit hyperactivity disorder and other disruptive disorders. ${ }^{59}$

Our results indicate that both quetiapine and olanzapine may have an incisive effect on impulsivity as measured by the IRS. In fact, olanzapine and quetiapine both act as antagonist at the 5-HT2A receptors, increasing noradrenergic firing activity ${ }^{60}$ which in turn increases 5-HT firing activity, ${ }^{61}$ thus reversing the impulsivity that is characterized by low 5 -HT activity (see Introduction).

In this trial, the equivalent doses of olanzapine $(18.5 \mathrm{mg})$ were relatively higher compared to those of quetiapine $(525 \mathrm{mg})$, the latter not reaching optimal anti-psychotic and likely anti-aggression efficacy. However, our results suggest that even at lower doses, quetiapine was effective in decreasing psychotic symptoms and improving depression items in schizophrenic patients.

In this study, we have noticed that MOAS might not be a sensitive enough scale in a high-security environment where patients are strictly watched and prevented from committing aggressive acts against themselves and other people. In fact, MOAS items at baseline were very low considering this type of patient; nevertheless, we were able to detect episodes of verbal aggression and aggression against objects, but not physical aggression or aggression against self and others. Finally, considering that impulsivity is one of the main causes of aggressive acts, ${ }^{2,3}$ the fact that both quetiapine and olanzapine decreased impulsive behavior can be considered as a positive outcome for long-term treatment of aggression, independently from the effects on the MOAS. Other than decreases in impulsivity and psychotic symptoms, it is worthy to mention that this pilot study showed that quetiapine, but not olanzapine, improved the depressive symptoms in the sub-items of PANSS and BPRS. As mentioned in the Materials and Methods section, this study was carried out before the studies on the antidepressant effects of quetiapine were published ${ }^{62}$ thus confirming that these results were not biased by the clinical judgments of raters. The neurobiological mechanism explaining this difference may lie in the partial agonist activity of quetiapine for the 5-HT1A receptors and in the NE reuptake pump affinity of the metabolite norquetiapine; remarkably, these two pharmacological features are not shared by olanzapine. ${ }^{31}$ Our results suggest that quetiapine, rather than olanzapine, should be the first choice in schizophrenic patients with depressive symptomatology.

Finally, in our study, we did not notice significant differences at the endpoint in glucose, cholesterol, or triglycerides (except for one patient in the quetiapine group and another patient in the olanzapine group) as reported in other studies, ${ }^{63}$ but this lack of secondary effects on metabolism could be due to the relatively short duration of the clinical trial.

In conclusion, this study shows that olanzapine and quetiapine equally decrease impulsivity and improve psychotic symptoms in people suffering from schizophrenia and violent behavior, and that these two treatments have a distinct effect on depression, suggesting that atypical antipsychotics, because of their different receptorial affinities, may modulate the range of psychotic symptoms in different ways.

Because of the limited number of patients in the protocol, all the results and statements presented in this research need further extended double-blind studies in order to be confirmed and the presence of a positive $P$-value could simply be due to chance alone (type I error). Specifically, more trials are needed to further characterize the effects of atypical antipsychotics in specific clinical subpopulations and to evaluate their efficacy considering the type of symptomatology.

\section{Acknowledgments}

The authors thank Rita Bhatti for clinical assistance in the study and Mr Ian Debonnel, Dr Michael Tau, and Dr Francis Rodriguez Bambico for helping with the data bank and preliminary statistics. The authors thank the patients, psychiatrists, nurses, and all the 
staff of the "Institute Pinel de Montreal" for their kind and patient collaboration in the study.

\section{Disclosure}

This research was an investigator-initiated trial sponsored by AstraZeneca (Study code: 5077-9056, granted to GD); GG has received speaker honoraria from AstraZeneca Canada, Eli Lilly Canada Inc., and Merck Canada. The authors have no other conflicts of interest to declare.

\section{References}

1. Arseneault L, Moffitt TE, Caspi A, Taylor PJ, Silva PA. Mental disorders and violence in a total birth cohort - results from the Dunedin study. Arch Gen Psychiatry. 2000;57(10):979-986.

2. Seroczynski AD, Bergeman CS, Coccaro EF. Etiology of the impulsivity/aggression relationship: genes or environment? Psychiatry Res. 1999;86(1):41-57.

3. Apter A, Plutchik R, van Praag HM. Anxiety, impulsivity and depressed mood in relation to suicidal and violent behavior. Acta Psychiatr Scand. 1993;87(1):1-5.

4. Comai S, Tau M, Gobbi G. The psychopharmacology of aggressive behavior: a translational approach: part 1: neurobiology. J Clin Psychopharmacol. 2012;32(1):83-94.

5. Nelson RJ, Chiavegatto S. Molecular basis of aggression. Trends Neurosci. 2001;24(12):713-719.

6. Gobbi G, Debonnel G. What is a recommended treatment for aggression in a patient with schizophrenia? J Psychiatry Neurosci. 2003;28(4): 320-320.

7. Lee R, Coccaro E. The neuropsychopharmacology of criminality and aggression. Can J Psychiatry. 2001;46(1):35-44.

8. Asberg M, Träskman L, Thorén P. 5-HIAA in cerebrospinal fluid. A biochemical suicide predictor? Arch Gen Psychiatry. 1976;33(10): $1193-1197$.

9. Brown GL, Goodwin FK, Ballenger JC, Goyer PF, Major LF. Aggression in humans correlates with cerebrospinal fluid amine metabolites. Psychiatry Res. 1979;1(2):131-139.

10. Linnoila M, Virkkunen M, Scheinin M, Nuutila A, Rimon R, Goodwin FK. Low cerebrospinal fluid 5-hydroxyindoleacetic acid concentration differentiates impulsive from nonimpulsive violent behavior. Life Sci. 1983;33(26):2609-2614.

11. Virkkunen M, Goldman D, Nielsen DA, Linnoila M. Low brain serotonin turnover rate (low CSF 5-HIAA) and impulsive violence. J Psychiatry Neurosci. 1995;20(4):271-275.

12. Cremniter D, Jamain S, Kollenbach K, et al. CSF 5-HIAA levels are lower in impulsive as compared to nonimpulsive violent suicide attempters and control subjects. Biol Psychiatry. 1999;45(12):1572-1579.

13. Roggenbach J, Muller-Oerlinghausen B, Franke L. Suicidality, impulsivity and aggression - is there a link to 5HIAA concentration in the cerebrospinal fluid? Psychiatry Res. 2002;113(1-2):193-206.

14. Cleare AJ, Bond AJ. The effect of tryptophan depletion and enhancement on subjective and behavioral aggression in normal-male subjects. Psychopharmacology. 1995;118(1):72-81.

15. LeMarquand DG, Benkelfat C, Pihl RO, Palmour RM, Young SN. Behavioral disinhibition induced by tryptophan depletion in nonalcoholic young men with multigenerational family histories of paternal alcoholism. Am J Psychiatry. 1999;156(11):1771-1779.

16. LeMarquand DG, Pihl RO, Young SN, et al. Tryptophan depletion, executive functions, and disinhibition in aggressive, adolescent males. Neuropsychopharmacology. 1998;19(4):333-341.

17. Rogers RD, Blackshaw AJ, Middleton HC, et al. Tryptophan depletion impairs stimulus-reward learning while methylphenidate disrupts attentional control in healthy young adults: implications for the monoaminergic basis of impulsive behaviour. Psychopharmacology. 1999;146(4):482-491.
18. Bellus SB, Stewart D, Kost PP. Clozapine in aggression. Psychiatr Serv. 1995;46(2):187-187.

19. Buckley P, Bartell J, Donenwirth K, Lee S, Torigoe F, Schulz SC. Violence and schizophrenia: clozapine as a specific antiaggressive agent. Bull Am Acad Psychiatry Law. 1995;23(4):607-611.

20. Chengappa KNR, Ebeling T, Kang JS, Levine J, Parepally H. Clozapine reduces severe self-mutilation and aggression in psychotic patients with borderline personality disorder. J Clin Psychiatry. 1999;60(7): 477-484.

21. Fava M. Psychopharmacologic treatment of pathologic aggression. Psychiatr Clin North Am. 1997;20(2):427-451.

22. Glazer WM, Dickson RA. Clozapine reduces violence and persistent aggression in schizophrenia. J Clin Psychiatry. 1998;59:8-14.

23. Pabis DJ, Stanislav SW. Pharmacotherapy of aggressive behavior. Ann Pharmacother. 1996;30(3):278-287.

24. Rabinowitz J, Avnon M. Effect of clozapine on physical and verbal aggression. Schizophr Res. 1996;18(2-3):249-255.

25. Ratey JJ, Leveroni C, Kilmer D, Gutheil C, Swartz B. The effects of clozapine on severely aggressive psychiatric inpatients in a state hospital. J Clin Psychiatry. 1993;54(6):219-223.

26. Spivak B, Mester R, Wittenberg N, Maman Z, Weizman A. Reduction of aggressiveness and impulsiveness during clozapine treatment in chronic neuroleptic-resistant schizophrenic patients. Clin Neuropharmacol. 1997;20(5):442-446.

27. Volavka J, Zito JM, Vitrai J, Czobor P. Clozapine effects on hostility and aggression in schizophrenia. J Clin Psychopharmacol. 1993;13(4):287-289.

28. Wilson WH. Clinical review of clozapine treatment in a state hospital. Hosp Community Psychiatry. 1992;43(7):700-703.

29. Alvir JMJ, Lieberman JA, Safferman AZ, Schwimmer JL, Schaaf JA. Clozapine-induced agranulocytosis. Incidence and risk-factors in the United States. N Engl J Med. 1993;329(3):162-167.

30. Bymaster FP, Calligaro DO, Falcone JF, et al. Radioreceptor binding profile of the atypical antipsychotic olanzapine. Neuropsychopharmacology. 1996;14(2):87-96.

31. Fulton B, Goa KL. Olanzapine. A review of its pharmacological properties and therapeutic efficacy in the management of schizophrenia and related psychoses. Drugs. 1997;53(2):281-298.

32. Kinon BJ, Stauffer VL, Kollack-Walker S, Chen L, Sniadecki J. Olanzapine versus aripiprazole for the treatment of agitation in acutely ill patients with schizophrenia. J Clin Psychopharmacol. 2008;28(6): 601-607.

33. Krakowski MI, Czobor P, Citrome L, Bark N, Cooper TB. Atypical antipsychotic agents in the treatment of violent patients with schizophrenia and schizoaffective disorder. Arch Gen Psychiatry. 2006;63(6):622-629.

34. Strous RD, Kupchik M, Roitman S, et al. Comparison between risperidone, olanzapine, and clozapine in the management of chronic schizophrenia: a naturalistic prospective 12-week observational study. Hum Psychopharmacol. 2006;21(4):235-243.

35. Volavka J, Czobor P, Nolan K, et al. Overt aggression and psychotic symptoms in patients with schizophrenia treated with clozapine, olanzapine, risperidone, or haloperidol. J Clin Psychopharmacol. 2004;24(2):225-228.

36. Newman-Tancredi A, Gavaudan S, Conte C, et al. Agonist and antagonist actions of antipsychotic agents at 5-HT1A receptors: a [S-35]GTPgammaS binding study. Eur J Pharmacol. 1998;355(2-3):245-256.

37. Tyson PJ, Roberts KH, Mortimer AM. Are the cognitive effects of atypical antipsychotics influenced by their affinity to 5HT-2A receptors? Int J Neurosci. 2004;114(6):593-611.

38. Rauser L, Savage JE, Meltzer HY, Roth BL. Inverse agonist actions of typical and atypical antipsychotic drugs at the human 5-hydroxytryptamine(2C) receptor. J Pharmacol Exp Ther. 2001;299(1): $83-89$.

39. Arango C, Bernardo M. The effect of quetiapine on aggression and hostility in patients with schizophrenia. Hum Psychopharmol. 2005;20(4):237-241. 
40. Cantillon M, Goldstein JM. Quetiapine fumarate reduces aggression and hostility in patients with schizophrenia. Poster presented at: American Psychiatric Association 151st Annual Meeting; May 30-June 4, 1998; Toronto, Canada.

41. Chengappa KN, Goldstein JM, Greenwood M, John V, Levine J. A post hoc analysis of the impact on hostility and agitation of quetiapine and haloperidol among patients with schizophrenia. Clin Ther. 2003;25(2): 530-541.

42. Kalali A, Schulz SC, Kahn RS, et al. Effectiveness of once-daily extended release quetiapine fumarate (quetiapine $\mathrm{xr}$ ) for excitability, hostility and aggression in schizophrenia. Schizophr Res. 2008; 98 Suppl 1:164.

43. Villari V, Rocca P, Fonzo V, Montemagni C, Pandullo P, Bogetto F. Oral risperidone, olanzapine and quetiapine versus haloperidol in psychotic agitation. Prog Neuropsychopharmacol Biol Psychiatry. 2008;32(2):405-413.

44. Bitter I, Czobor P, Dossenbach M, Volavka J. Effectiveness of clozapine, olanzapine, quetiapine, risperidone, and haloperidol monotherapy in reducing hostile and aggressive behavior in outpatients treated for schizophrenia: a prospective naturalistic study (IC-SOHO). Eur Psychiatry. 2005;20(5-6):403-408.

45. Swanson JW, Swartz MS, Van Dorn RA, et al. Comparison of antipsychotic medication effects on reducing violence in people with schizophrenia. Br J Psychiatry. 2008;193(1):37-43.

46. Foley SR, Kelly BD, Clarke M, et al. Incidence and clinical correlates of aggression and violence at presentation in patients with first episode psychosis. Schizophr Res. 2005;72(2-3):161-168.

47. Lecrubier Y, Braconnier A, Said S, Payan C. The impulsivity rating scale (IRS): preliminary results. Eur Psychiatry. 1995;10(7):331-338.

48. Overall JE GD. The brief psychiatric rating scale. Psychol Rep. 1962;10: 799-812.

49. Kay SR, Fiszbein A, Opler LA. The positive and negative syndrome scale (PANSS) for schizophrenia. Schizophr Bull. 1987;13(2):261-276.

50. Guy W. ECDEU Assessment Manual for Psychopharmacology Revised, 1976. Rockville, MA: US Department of Health, Education, and Welfare; 1976:218-222.

51. Streiner D GJ. Intention to treat analysis in clinical trials when there are missing data. Evid Based Ment Health. 2001;4:70-71.

52. Breier A, Meehan K, Birkett M, et al. A double-blind, placebo-controlled dose-response comparison of intramuscular olanzapine and haloperidol in the treatment of acute agitation in schizophrenia. Arch Gen Psychiatry. 2002;59(5):441-448.
53. Lambert M, Huber CG, Naber D, et al. Treatment of severe agitation with olanzapine in 166 patients with schizophrenia, schizoaffective, or bipolar I disorder. Pharmacopsychiatry. 2008;41(5):182-189.

54. San L, Arranz B, Querejeta I, Barrio S, De la Gandara J, Perez V. A naturalistic multicenter study of intramuscular olanzapine in the treatment of acutely agitated manic or schizophrenic patients. Eur Psychiatry. 2006;21(8):539-543.

55. Wang X, Savage R, Borisov A, et al. Efficacy of risperidone versus olanzapine in patients with schizophrenia previously on chronic conventional antipsychotic therapy: a switch study. J Psychiatr Res. 2006;40(7):669-676.

56. Wright P, Birkett M, David SR, et al. Double-blind, placebo-controlled comparison of intramuscular olanzapine and intramuscular haloperidol in the treatment of acute agitation in schizophrenia. Am J Psychiatry. 2001;158(7):1149-1151.

57. Ganesan S, Levy M, Bilsker D, Khanbhai I. Effectiveness of quetiapine for the management of aggressive psychosis in the emergency psychiatric setting: a naturalistic uncontrolled trial. Int J Psychiatry Clin Pract. 2005;9(3):199-203.

58. Kim E, Bijlani M. A pilot study of quetiapine treatment of aggression due to traumatic brain injury. $J$ Neuropsychiatry Clin Neurosci. 2006;18(4):547-549.

59. Kronenberger WG, Giauque AL, Lafata DE, Bohnstedt BN, Maxey LE, Dunn DW. Quetiapine addition in methylphenidate treatment-resistant adolescents with comorbid ADHD, conduct/oppositional-defiant disorder, and aggression: a prospective, open-label study. $J$ Child Adolesc Psychopharmacol. 2007;17(3):334-347.

60. Dawe GS, Huff KD, Vandergriff JL, Sharp T, O’Neill MJ, Rasmussen K Olanzapine activates the rat locus coeruleus: in vivo electrophysiology and c-Fos immunoreactivity. Biol Psychiatry. 2001;50(7):510-520.

61. Blier P, Szabo ST. Potential mechanisms of action of atypical antipsychotic medications in treatment-resistant depression and anxiety. J Clin Psychiatry. 2005;66 Suppl 8:30-40.

62. Calabrese JR, Keck PE Jr, Macfadden W, et al. A randomized, double-blind, placebo-controlled trial of quetiapine in the treatment of bipolar I or II depression. Am J Psychiatry. 2005;162(7):1351-1360.

63. Atmaca M, Kuloglu M, Tezcan E, Gecici O, Ustundag B. Weight gain, serum leptin and triglyceride levels in patients with schizophrenia on antipsychotic treatment with quetiapine, olanzapine and haloperidol. Schizophr Res. 2003;60(1):99-100.
Neuropsychiatric Disease and Treatment

\section{Publish your work in this journal}

Neuropsychiatric Disease and Treatment is an international, peerreviewed journal of clinical therapeutics and pharmacology focusing on concise rapid reporting of clinical or pre-clinical studies on a range of neuropsychiatric and neurological disorders. This journa is indexed on PubMed Central, the 'PsycINFO' database and CAS

\section{Dovepress}

The manuscript management system is completely online and includes a very quick and fair peer-review system, which is all easy to use. Visit http://www.dovepress.com/testimonials.php to read real quotes from published authors. 